ARTICLE

DOI: $10.1038 / \mathrm{s} 41467-017-01001-9$

\title{
Characteristic rotational behaviors of rod-shaped cargo revealed by automated five-dimensional single particle tracking
}

\author{
Kuangcai Chen (10) ${ }^{1,2}$, Yan Gu ${ }^{2,4}$, Wei Sun ${ }^{2,5}$, Bin Dong ${ }^{1,2}$, Gufeng Wang (iD ${ }^{2,6}$, Xinxin Fan ${ }^{3}$,
} Tian $\mathrm{Xia}^{3} \&$ Ning Fang (1) ${ }^{1}$

We report an automated single particle tracking technique for tracking the $x, y, z$ coordinates, azimuthal and elevation angles of anisotropic plasmonic gold nanorod probes in live cells. These five spatial coordinates are collectively referred to as 5D. This method overcomes a long-standing challenge in distinguishing rotational motions from translational motions in the $z$-axis in differential interference contrast microscopy to result in full disclosure of nanoscale motions with high accuracy. Transferrin-coated endocytic gold nanorod cargoes initially undergo active rotational diffusion and display characteristic rotational motions on the membrane. Then as the cargoes being enclosed in clathrin-coated pits, they slow down the active rotation and experience a quiet period before they restore active rotational diffusion after fission and eventually being transported away from the original entry spots. Finally, the $3 \mathrm{D}$ trajectories and the accompanying rotational motions of the cargoes are resolved accurately to render the intracellular transport process in live cells.

\footnotetext{
${ }^{1}$ Department of Chemistry, Georgia State University, Atlanta, GA 30303, USA. ${ }^{2}$ Department of Chemistry, lowa State University, Ames, IA 50011, USA. ${ }^{3}$ Department of Electronics and Information Engineering, Huazhong University of Science and Technology, Wuhan 430074, China. ${ }^{4}$ Present address: The Bristol-Myers Squibb Company, Devens, MA 01434, USA. ${ }^{5}$ Present address: Corning Inc., Painted Post, NY 14870, USA. ${ }^{6}$ Present address: Department of Chemistry, North Carolina State University, Rayleigh, NC 27695, USA. Kuangcai Chen, Yan Gu and Wei Sun contributed equally to this work.

Correspondence and requests for materials should be addressed to T.X. (email: tianxia@hust.edu.cn) or to N.F. (email: nfang@gsu.edu)
} 
S ingle particle tracking (SPT) has become an indispensable approach in our attempts to understand the detailed working mechanisms of biomolecules in complex cellular environments ${ }^{1}$. Motions of single imaging probes recorded in SPT experiments contain rich information, including the $x, y, z$ coordinates and the two orientation angles (azimuthal angle $\phi$ and elevation angle $\psi$, as defined in Fig. 1a) of the probe's transition dipole over a series of time steps. After two decades of development, lateral localization of single imaging probes with nanometer accuracy ${ }^{2-4}$ in the $(x, y)$ plane has become routine, and many strategies have also been developed to improve the $z$-tracking ability ${ }^{5-18}$. However, the ability to acquire accurate measurements in all five spatial parameters $(x, y, z, \phi, \psi)$ simultaneously remains elusive. These five coordinates are collectively referred to as five dimensions (5D) in the present study.

The challenges are mainly two-fold. First, it is difficult to resolve the dipole orientation of single fluorescent probes in a cellular environment with reasonably fast temporal resolution due to the well-known limitations, such as high background and fast photobleaching. This challenge can be circumvented by using anisotropic plasmonic gold nanorods as alternative rotational tracking probes. The exceptionally high absorption and scattering cross-sections of gold nanorods result in strong and stable signals at the localized surface plasmon resonance wavelengths ${ }^{19}$. We have previously developed the single particle orientation and rotational tracking (SPORT) technique for direct visualization of rotational dynamics of gold nanorods in differential interference contrast (DIC) microscopy at millisecond temporal resolution, while still obtaining high-contrast images of cellular features ${ }^{20-23}$. Relatively low illumination light intensity from a typical halogen lamp, instead of a stronger laser light source ${ }^{24}$ brings minimal disruptions to cell functions, making it particularly suitable for long-term SPT experiments in live cells. Polarized or defocused dark field (DF) microscopy ${ }^{25-27}$ is another viable choice for tracking single plasmonic gold nanoparticles, although its application to SPT in live cells is more challenging due to the generally high-scattering background in the cellular environments. In addition, the two-photon luminescence ${ }^{28}$ and photothermal heterodyne imaging ${ }^{29}$ can also image gold nanorods; however, the relatively high-power laser light source and generally slow rasterscanning process can limit their applicability in single cell imaging. Furthermore, significant progresses have also been made in measuring gold nanorods in vivo ${ }^{30-32}$; however, these in vivo methods were not suitable for disclosing the motions of individual gold nanorods due to insufficient sensitivity or speed.

The second challenge is associated with the difficulties to distinguish rotational motions of the anisotropic probe from its movement in the $z$-axis because both types of motion result in similar changes in signal in DIC microscopy. This ambiguity would result in incorrect assignment of the dipole's orientation.

Herein we introduce an automated five-dimensional single particle tracking (5D-SPT) technique to overcome these challenges. The core idea is to combine the DIC microscopy-based SPORT technique with the principle of parallax microscopy ${ }^{15}, 16$ for simultaneous spatial and rotational tracking. Parallax is a mechanism for humans to gain depth perception by creating a displacement in the apparent position of an object viewed in two eyes. It is worth noting that the dependence of the defocused DF image patterns on the probe's relative axial distance to the focal plane can be utilized for axial localization ${ }^{28,33}$; however, the complexity of the defocused image patterns and relatively weak defocused signal intensities lead to low axial localization accuracy and precision, as well as small vertical tracking distance.

The 5D-SPT technique has been applied to disclose the characteristic motions of transferrin-coated gold nanorod cargos at each stage of the clathrin-mediated endocytosis (CME) pathway and subsequent intracellular transport. This technique makes significant improvements in our ability to detect the presence, location, orientation, translation, and rotation of nanoparticle probes in live cells. Other worth noting advantages include the simultaneous visualization of cellular features with high contrast and the ability to follow target probes for long durations (limited mainly by cell viability and computer data acquisition) and in large 3D volumes (within the camera's field of view and the travel distance of a high-precision piezo objective scanner). Using the 5D-SPT technique, the current study focuses full motions of cargos in live cells have been resolved with unprecedented details and accuracy.

\section{Results}

Design of parallax-DIC microscopy. The SPORT technique previously developed in our laboratory takes the advantage of the plasmon resonance-associated birefringence of gold nanorods ${ }^{25}, 27,29,34$ and Nomarski-type DIC microscopy 35,36 , which utilizes two orthogonally polarized beams for illumination. Optically anisotropic gold nanorods show as diffraction-limited spots with bright and dark peaks on a gray background in DIC images (Supplementary Fig. 1). The bright and dark intensities are independent measures of the effective projections of the nanorods onto the two polarization axes, from which the $3 \mathrm{D}$ orientation of gold nanorods can be resolved in each DIC image $^{20,22,23}$. The normalized DIC bright and dark intensities traces can be readily converted to the orientation measurements.

The new 5D-SPT technique uses parallax to sense the axial movement of the target object, and at the same time, employs an automatic feedback loop algorithm to control the focal plane of the objective and bring the target object back to focus repetitively. In such an implementation, the target object is kept in focus to provide the highest possible $\mathrm{S} / \mathrm{N}$ for high-accuracy $2 \mathrm{D}$ localization and orientation determination, while the axial $(z)$ positions are recorded as the positions of the high-precision objective scanner.

Parallax is realized on an upright DIC microscope by inserting a wedge prism at the back focal plane of the objective (Fig. 2). Half of the light that has passed through the sample maintains the original path, while the other half of the light is deviated slightly by the wedge prism. Two images of the same sample are formed in the upper and lower halves of the sensor area. These images will be referred to as "half-plane" images in the rest of the discussion because each image utilizes half of the numerical aperture of the objective, while conventional microscopy images utilizing the full numerical aperture will be referred to as "full-plane" images.

The movement of a target object in the axial $(z)$ direction is resolved by monitoring the distance $(d)$ between the pair of halfplane images of the same object. For any object in the focal plane, the distance between its two half-plane images $\left(d_{0}\right)$ is a fixed value determined by the deviation angle of the wedge prism. When the target object moves upwards $(\Delta z>0)$ towards the objective, the two half-plane images move away from one another to increase $d$. When the target object moves downwards $(\Delta z<0)$ away from the objective, the two images move toward one another to shorten $d$. Supplementary Movie 1 shows parallax-DIC images of a stationary gold nanorod at three orientations traveling smoothly from $-0.4 \mu \mathrm{m}$ below the focal plane to $+0.4 \mu \mathrm{m}$ above the focal plane at a speed of $100 \mathrm{~nm}$ per second with a single frame exposure time of $200 \mathrm{~ms}$. The nanorod shows orientationdependent DIC images (Fig. 1b).

The parallax-DIC microscope inherits the conventional DIC microscope's ability of visualizing the cell morphology and detailed intracellular structures. Supplementary Fig. 2 shows the two half-plane images of a live cell at different vertical sections. 
Localization in parallax-DIC microscopy. Localization in parallax-DIC microscopy is essentially a task of finding the accurate positions of the pair of half-plane images. Gaussian fitting, the most widely used localization method, is not suitable for DIC microscopy because the anti-symmetric DIC point spread function (PSF), which consists of apposed bright and dark a

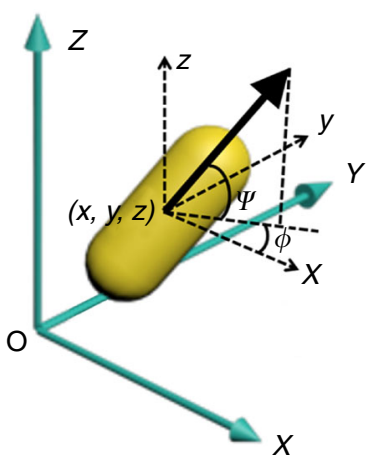

b

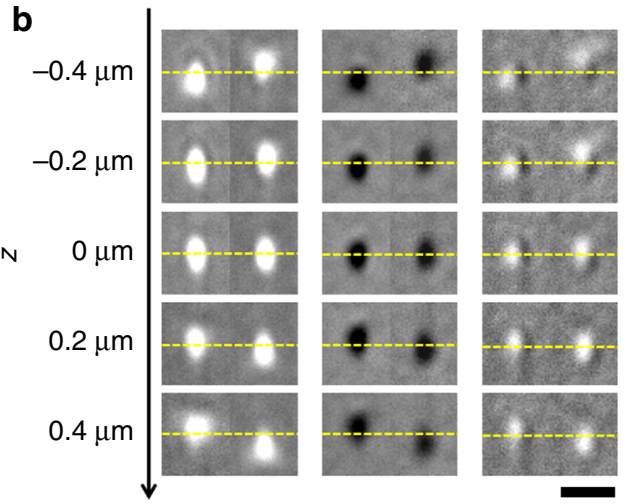

C

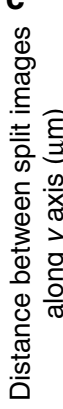

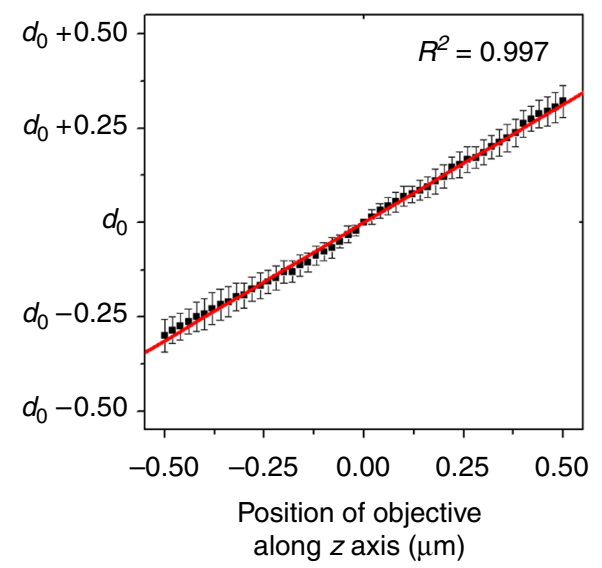

d

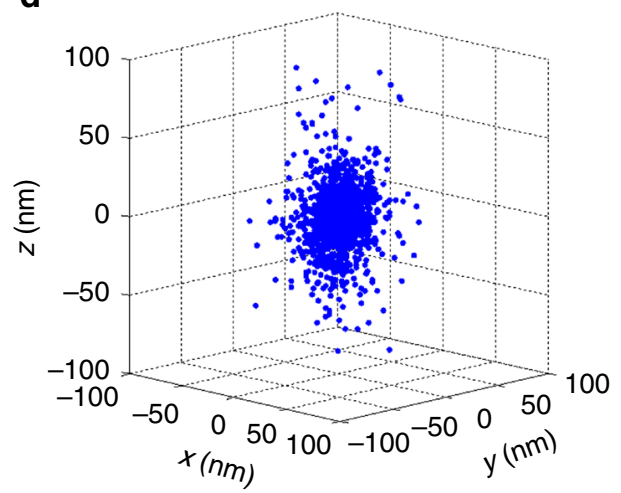

e

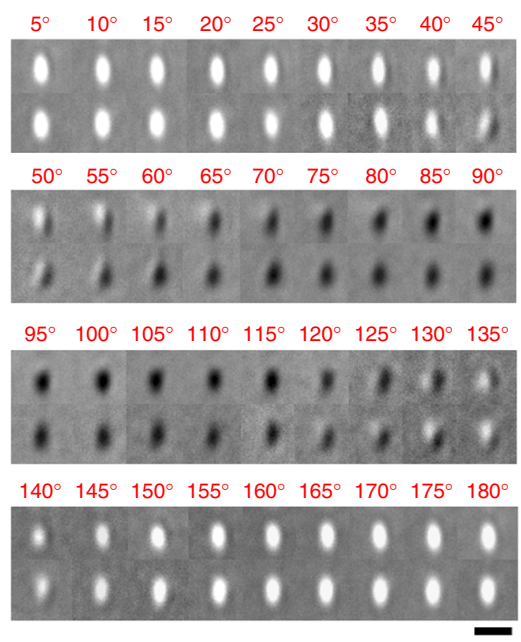

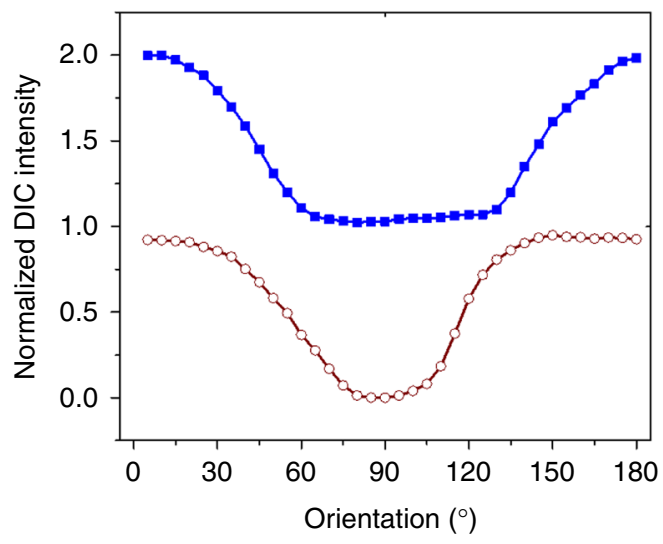

Fig. 1 Principle and localization precision of the parallax-DIC microscope. a Schematic illustration of a gold nanorod placed in the 3D coordinate system. The $x$ - and $y$-axes are set according to the polarization directions of the illumination light. The dipole shown here corresponds to the long axis of the gold nanorod, whose centroid locates at $(x, y, z)$. The azimuthal angle and the elevation angle of the longitudinal axis of the gold nanorod are presented as $\phi$ and $\psi$, respectively. b Bright (left), dark (middle), and half-bright/half-dark (right) images of a gold nanorod $(40 \times 80 \mathrm{~nm})$ at different orientations and vertical positions. The half-plane images are aligned by the nanorod's center of mass at the focal plane $(z=0 \mu \mathrm{m})$. The yellow dashed lines indicate the $z$-position of the center of the gold nanorod in focus. Scale bar is $1 \mu \mathrm{m}$. c Calibration of the 5D-SPT technique. The distance between the two half-plane images changes linearly with the $z$-position of the gold nanorod within $\pm 0.5 \mu \mathrm{m}$ of the focal plane. The distance for an in-focus gold nanorod is defined as $d_{0}$. The error bars reflect the standard deviations of the distances measured when the nanorod's azimuthal angle $\phi$ changes in the range of $0-180^{\circ}$ with $5^{\circ}$ steps (error bar \pm s.d., $n=36$ ). The calibration curve returned a slope of $+0.626(d / \Delta z)$ and $R^{2}$ of 0.997 . $\mathbf{d}$ Three-dimensional localization distribution of the gold nanorod with standard deviations of $11 \mathrm{~nm}$ in $x, 14 \mathrm{~nm}$ in $y$, and $17 \mathrm{~nm}$ in $z$. e The in-focus half-plane image patterns of a gold nanorod at different azimuthal angles when the sample slide is rotated in $5^{\circ}$ steps for $180^{\circ}$. The scale bar represents $1 \mu \mathrm{m}$. f The normalized bright part (blue) and dark part (brown) intensities of the half-plane images on the top in each pair in $\mathrm{E}$ 
a

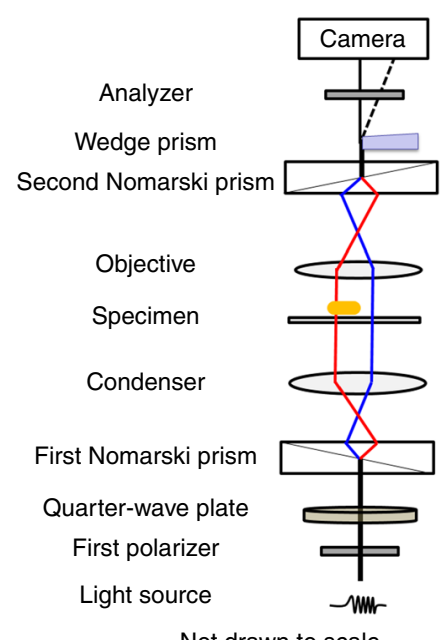

b

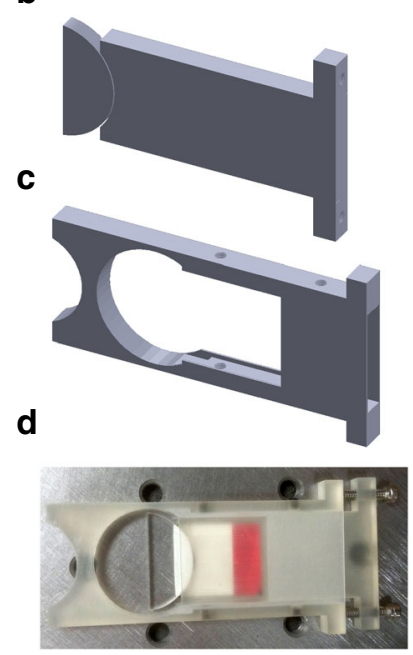

e

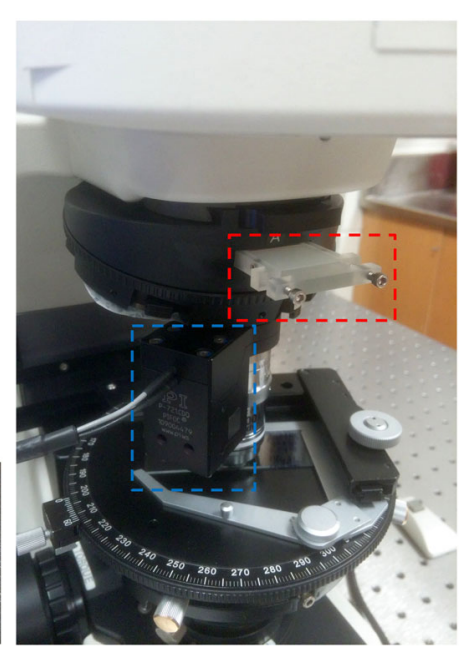

Fig. 2 Parallax-DIC microscope and 5D-SPT instrumental set-up. a Schematic illustration of the optical path in parallax-DIC microscope. High-precision objective scanner was not drawn in the scheme. $\mathbf{b}$ Model of the insert mounted with the custom-cut wedge prism. c Model of the slot. $\mathbf{d}$ Photograph of the wedge prism set installed on the 3D-printed insert housing in the 3D-printed slot. The position of the insert and wedge prism were optimized and fixed by four \#4/40 ×1/8 socket set screws and two \#4/40 ×1/2 hex socket head cap screws to split the light in half at the back focal plane of the objective. e Photograph of the 5D-SPT instrumental set-up. The optimized wedge prism set was insert to the light path (as indicated in the red dashed box) to enable the parallax-DIC mode and the objective scanner (as shown in the blue dashed box) was use to deploy the automated 5D-SPT

portions over a gray background, cannot be fitted with a simple mathematical function. Therefore, the more complicated modelbased correlation mapping procedures are required for localizing particle probes in DIC microscopy ${ }^{37,38}$. Localization in parallaxDIC microscopy is even more challenging as the half-plane PSF appears to be stretched in the direction perpendicular to the split plane, as illustrated in the simulated "half-plane" images (Supplementary Fig. 3).

To overcome the complications in the parallax-DIC PSF, we have implemented a redesigned correlation mapping procedure, in which one of the half-plane images is used as the model to map the other image in order to find the centers of these two images. With the wedge prism splitting the light in half, the two resulting half-plane PSFs appear to be the mirror image of each other. The correlation maps of the two half-plane images with correlation scores above a set threshold (typically $0.4-0.6$ ) are weighed to find their relative coordinates. The distance between the two halfplane images is thus determined and can be translated into the axial position of the target probe. The axial localization is calibrated by scanning the focal plane of the objective vertically in $20 \mathrm{~nm}$ steps through a stationary gold nanorod and correlating the distance between the two images with the axial position of the sample (Fig. 1c).

Evaluation of 5D-SPT. The implementation (Fig. 2) and evaluation of the 5D-SPT technique are discussed in detail in the Supplementary Note 1 . The figures of merit are summarized in this section.

It is important to strike an ideal balance among temporal, spatial, and angular resolution in 5D-SPT experiments. These three types of resolving power are competing for the same pool of photons. As the temporal resolution increases, the noise level in the measured bright and dark DIC intensities increases, resulting in less accurate assessment of rotation rate and mode.

The response time of the objective scanner and the speed of its interface to the computer determine the fastest temporal resolution of 5D-SPT technique to be $\sim 1 \mathrm{~ms}$. A series of parallaxDIC images taken at different exposure times are displayed in
Supplementary Fig. 4. To ensure sufficiently high-angular resolution and localization precision in live cells, the $40 \times 80 \mathrm{~nm}$ gold nanorod sample and the exposure time of $33 \mathrm{~ms}$ were employed in most of the 5D-SPT experiments reported in this paper, unless otherwise noted. The precision measurements of the 3D localization yield standard deviations of 11,14 , and $17 \mathrm{~nm}$ in the $x, y$, and $z$ axes, respectively (Fig. $1 \mathrm{~d}$ and Supplementary Fig. 5). The evaluation of the autofocusing under the parallaxDIC microscope is shown in Supplementary Fig. 6.

Finally, a set of parallax-DIC images of in-focus gold nanorod at different azimuthal angles is displayed in Fig. 1e. The bright and dark DIC intensities from each image are plot against the azimuthal angle in Fig. 1f. The orientation-dependent DIC intensities allow the orientation measurements.

Tracking endocytic nanocargos in 5D-SPT. Using the 5D-SPT technique, the rotational motions of transferrin-coated gold nanorods on the cell membrane and during the subsequent endocytosis by A549 human lung cancer cells were continuously followed. Transferrin ${ }^{39}$ molecules were conjugated to gold nanorod surface through PEG linker molecules to facilitate the cellular uptake ${ }^{21}$. Previous studies showed that CME plays a key role in cellular uptake of transferrin-coated gold nanospheres and nanorods of $\sim 50 \mathrm{~nm}$ in size ${ }^{40}$. In the present study, we visualized the dynamic internalization of transferrin-coated gold nanorods by transfected A549 human lung cancer cells to express enhanced yellow fluorescent protein (EYFP)-tagged clathrin ${ }^{41}$. The colocalization of gold nanorods and fluorescent bright spots from EYFP-clathrin gives direct evidence that CME is involved in the internalization of transferrin-coated gold nanorods. Supplementary Fig. 7 shows such a case where three out of eight transferrincoated gold nanorods binding to the membrane of a transfected A549 cell display co-localized EYFP fluorescence after a 30-min incubation in the cell culture medium containing the colloidal gold nanorods.

In addition, we observed that the internalization of transferrincoated gold nanorods was strongly dependent on the ambient temperature and osmotic pressure. After incubation at $4{ }^{\circ} \mathrm{C}$ for 
a

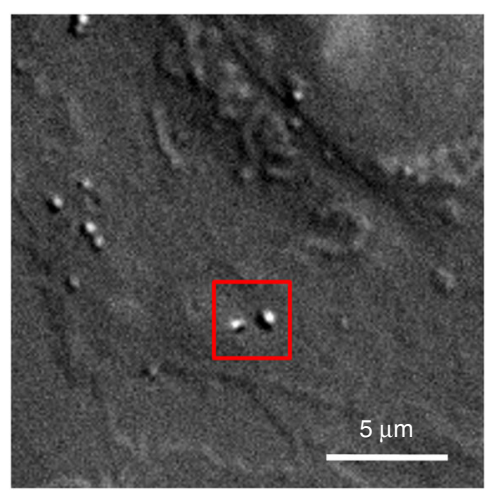

b

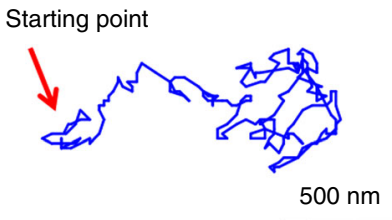

C

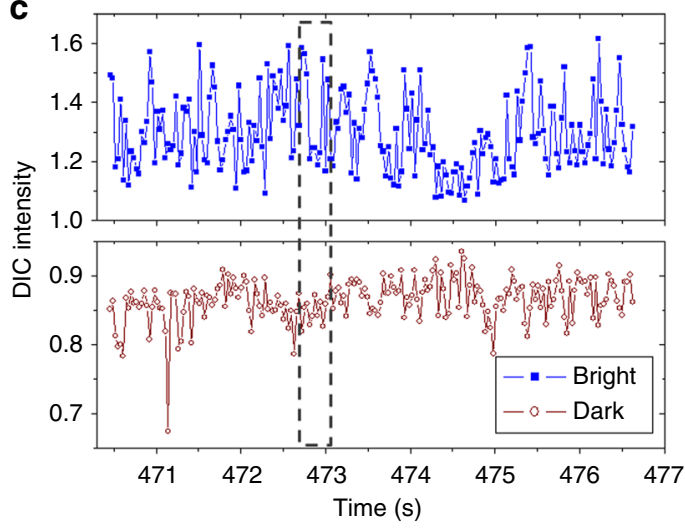

d

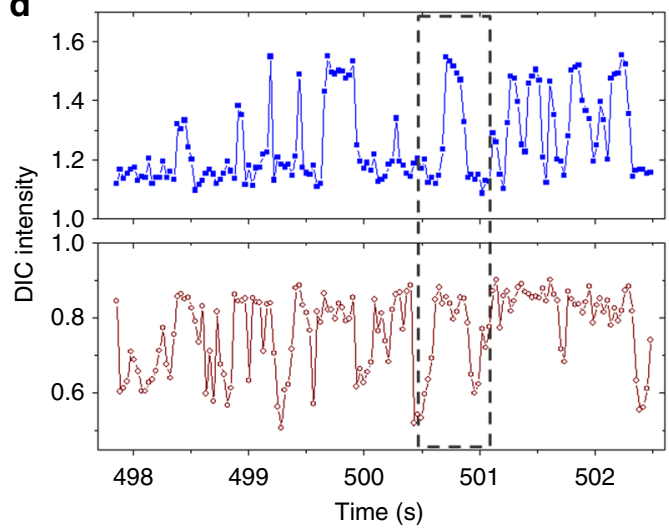

e

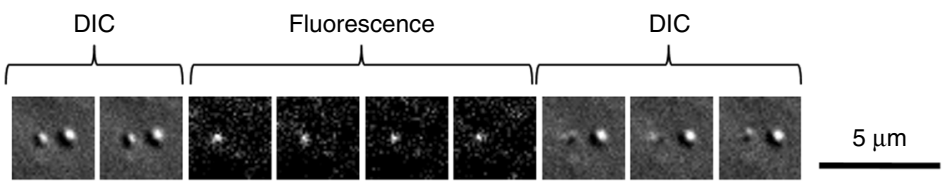

$t=530.8 \mathrm{~s}, \Delta t=310 \mathrm{~ms}$

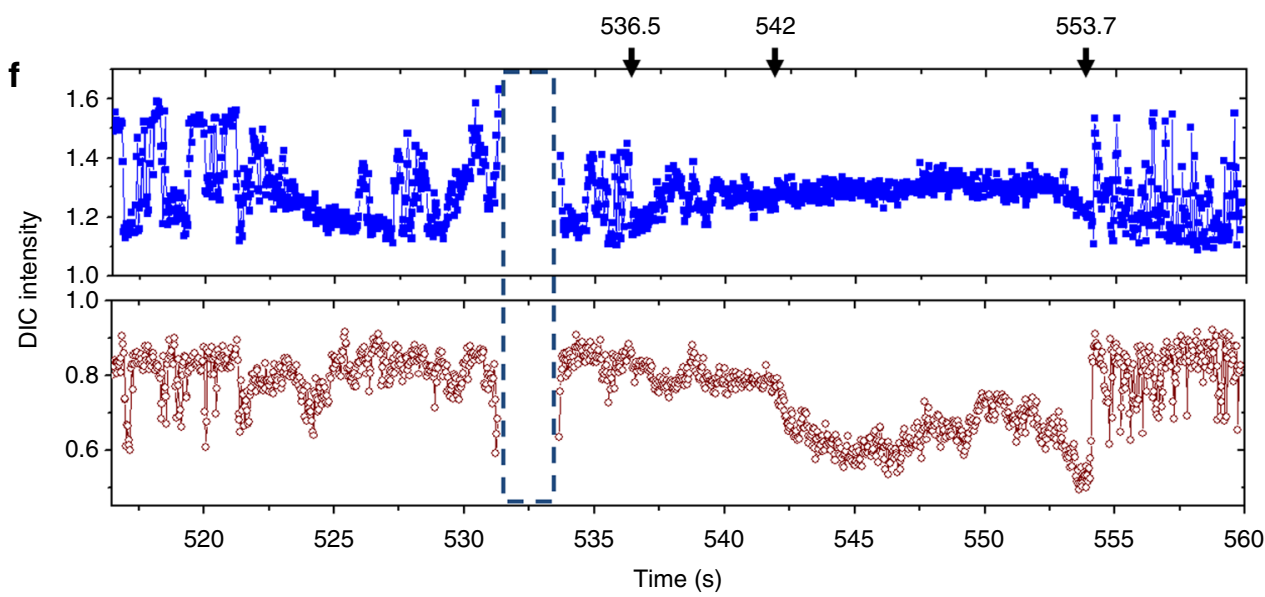

Fig. 3 A complete CME event of a gold nanorod by an A549 cell. Corresponding video are presented in Supplementary Movies 1-6. Time 0 is set to the moment when the nanorod first appeared on the membrane. a The cell and the location of the gold nanorod probe on the cell membrane. The nanorod (on the left in the red box) was beside a cell feature (on the right in the red box) that showed a constant contrast throughout the recording. $\mathbf{b}$ The trace of the nanorod's active translational movement at an early binding stage (Supplementary Movie 2). c DIC intensities of the nanorod in the same period as $\mathbf{b}$. The framed period shows typical out-of-plane rotations. d DIC intensities of the nanorod at a late stage (Supplementary Movie 3). The framed period shows typical in-plane rotations. e Sequential DIC and fluorescence images captured in tandem showing that the gold nanorod co-localized with a CCP (Supplementary Movie 4). The movie was acquired at 32 frames per second continuously and the displayed fluorescence images were the average of 10 consecutive images from the movie. $\mathbf{f}$ DIC intensities of the nanorod before and after scission of the nanorod-containing vesicle (Supplementary Movie 5). The blank period from 531.5 to $532.8 \mathrm{~s}$ was toggled to fluorescence mode in checking clathrin fluorescence. The nanorod lost active rotation at $536.5 \mathrm{~s}$ and regained rotational and translational freedoms at $553.7 \mathrm{~s}$. The same particle was observed to lose co-localization of fluorescence from EYFP-clathrin, indicating the clathrin disassembly (Supplementary Movie 6). Supplementary Movie 7 shows that the particle was actively transported at $45.7 \mathrm{~s}$ after scission in DIC mode 
$2 \mathrm{~h}$, a significant amount of gold nanorods were observed binding to the cell membrane, but not being endocytosed. After the temperature was raised to $37^{\circ} \mathrm{C}$, over half of the nanorods initially spotted on the cell membrane were internalized within the first $2 \mathrm{~h}$. In another control experiment, when the cells were treated with a hypertonic media (Sucrose) at $37^{\circ} \mathrm{C}$, significant decreases in the uptake of the nanorods within the first $2 \mathrm{~h}$ were observed, compared to the nanorod uptake by the cells not treated with the hypertonic media (Supplementary Fig. 8). The temperature dependence and the response to the hypertonic treatment, which disrupts the formation of clathrin-coated vesicles, are consistent with the previous reports that CME is the key pathway for the cellular uptake of nanometer-sized particles $40,42,43$.

Furthermore, specific mean to inhibit clathrin-mediated endocytosis was carried out by siRNA knockdown of AP-2 $\mu 2$ subunit in A549 cells. The siRNA oligonucleotide sequence 5'-GAGCAUGUGCACGCUGGCCA-3' targeting $\mu 2-2$ subunit of AP-2 is highly effective to achieve nearly complete depletion of AP-2 with two cycles of transfection, as reported by Motley et al. ${ }^{44}$ and confirmed by others ${ }^{45}, 46$. The siRNA treatment was performed following the established protocol. As shown in Supplementary Fig. 9, control cells showed much higher nanorod uptake compare to the cells subjected to AP-2 depletion.

In all the endocytosis 5D-SPT experiments, the parallax-DIC mode was activated intermittently (every few seconds) by inserting the wedge prism into the light path to compensate for focus drifting and keep the target gold nanorod in focus. During the remaining imaging time, the original DIC and fluorescence modes were operated alternately to acquire the orientation information of gold nanorods and the formation of fluorescent EYFP-clathrin coats.

Rotational pattern before endocytosis. We followed the dynamic uptake of each transferrin-coated gold nanorod continuously from its abrupt appearance on the cell membrane until the nanorod was linearly transported over long distances-convincing evidence of the nanorod being inside the cell. Unlike conventional SPT probes, gold nanorods are easy to identify, giving flickering images due to their own fast rotation on the cell membrane. More importantly, the nanorods display characteristic rotational motions at different binding and endocytic stages. Figure 3 shows such an example of a complete process from the nanorod landing on the cell membrane to being endocytosed by an A549 cell.

When the nanorod first fell on the cell membrane, it showed active lateral diffusion and fast rotation as revealed by the fluctuation of the DIC intensities (e.g., Fig. 3a-d and Supplementary Movies 2 and 3). The rotational motions of gold nanorods can be correlated to the recorded DIC intensity changes as discussed in details in our previous publications based on the correlation of computer-simulated results and experimental data $^{20,23}$. There are two fundamental rotation modes: the inplane rotation in which the rotation axis falls in the focal plane and the nanorod rotates within the plane, and the out-of-plane rotation in which the rotation axis is perpendicular to the focal plane and the nanorod rotates out of the plane. Distinctive changing DIC image patterns are associated with these two modes, with a correlated bright and dark intensity changes for the in-plane rotation mode and an anti-correlated bright and dark intensity changes for the out-of-plane rotation mode.

The motions of nanorod at the early stage of binding were characterized by active lateral diffusion in a relatively large area on the cell membrane (e.g., Fig. $3 b, c$ and Supplementary Movie 2) and a random mix of the in-plane and out-of-plane rotation modes as revealed by the DIC intensity changing patterns. For example, during the framed period (472.7-473.2 s) in Fig. $3 c$, the anti-correlated bright and dark intensity changes suggested that the nanorod likely swung around one of the polarization directions in an out-of-plane rotation mode.

The nanorod gradually lost its translational and rotational freedoms on the cell membrane. The lateral movement quickly became restrained and came to a complete stop at $497.9 \mathrm{~s}$. The rotational motion also became much slower, giving much less frequent fluctuations in the DIC intensities (Fig. 3d and Supplementary Movie 3). From the DIC intensities, we can identify that a component of in-plane rotation mode became more pronounced. For example, during the framed period (500.4-501.1 s) in Fig. 3d, the nanorod's DIC intensities changed in a correlation manner from completely dark to completely bright and then to completely dark again, showing a typical in-plane rotation mode.

The gradual loss of translational and rotational freedoms and the increase of the in-plane rotation component were an indication of the assembling of the endocytic machinery around the gold nanorod. A transient in-plane rotation may be an indication that the nanorod is tethered to the cell membrane by multiple binding sites. However, this kind of multi-point binding is highly dynamic and can dissociate at the next few milliseconds as indicated by the disappearance of the in-plane rotation mode.

Pearson's correlation coefficient analysis was applied to evaluate the rotational patterns. We have shown previously that for gold nanorod's in-plane rotation, the DIC bright and dark intensities are highly correlated with a correlation coefficient close to 1, while for the out-of-plane rotation, the DIC bright and dark intensities are anti-correlated with a correlation coefficient of $-1^{23}$. Supplementary Fig. 10 shows the correlation coefficients of the DIC bright and dark intensities of 150 transferrin-coated gold nanorods at different stages of interacting with the cell membrane. For the nanorods having active lateral diffusion in a relatively large area at the early stage of binding on the cell membrane, the distribution of correlation coefficient is centered at 0.48 with higher proportion of smaller correlation coefficients. This indicates the nanorods have higher tendency of out-of-plane motions due to the electrostatic repulsion between the negatively charged transferrin-coated gold nanorods and the negatively charged cell membrane. At the stage when gold nanorods lose their translational freedoms, the distribution of correlation coefficient is centered at 0.65 . With specific binding to cell membrane receptors, gold nanorods perform predominantly inplane rotation. The difference in the distributions of the correlation coefficients at different binding stages is statistically significant with a paired Student's $t$-test $(p<0.001)$.

Characteristic rotational motions during endocytosis. For all the nanorods that were eventually internalized, their fast rotational motions slowed down drastically at a certain point of time, an indication that the nanorods were enclosed in clathrin-coated pits (CCPs). The nanorods then all experienced a relatively quiet period of several tens of seconds, during which the nanorods showed no active rotational diffusion but rather slow, directed orientation changes, mostly likely under the influence of endocytic protein machinery. At the end of the quiet period, the nanorods restored active rotational diffusion, indicating the nanorod-containing vesicles were detached from the membrane.

This active rotation-quiet period-active rotation pattern was observed for all of the recorded $(>100)$ endocytosis cases. In the case shown in Fig. 3, the co-localization of the fluorescent CCP and the nanorod was observed at $530.8 \mathrm{~s}$ (Fig. 3e). Then the nanorod stopped active rotational diffusion at $\sim 536.5 \mathrm{~s}$, followed 

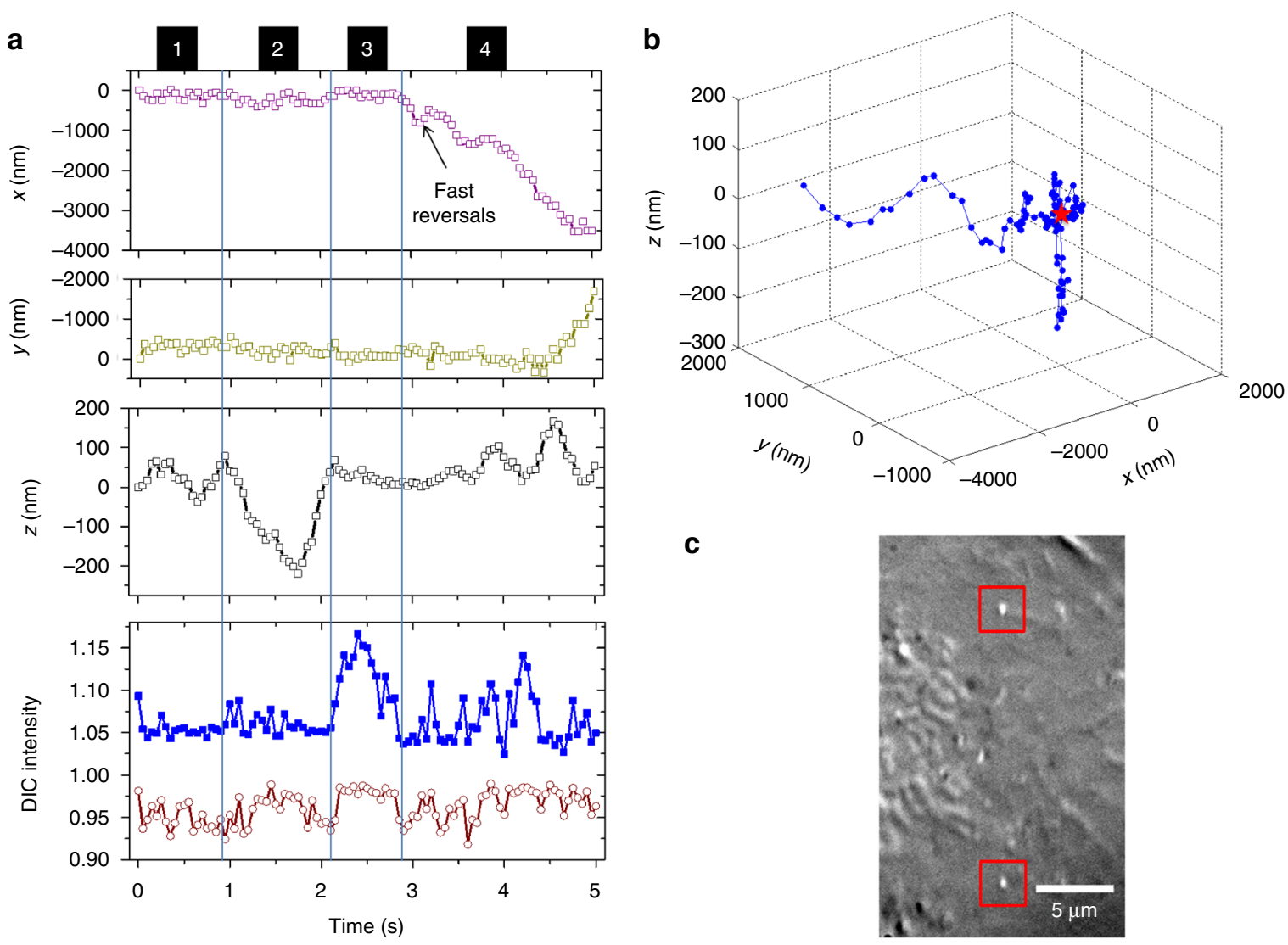

Fig. 4 Intracellular transport of a nanorod-containing vesicle in a living A549 cell. a The time series of the $x, y$, and $z$ displacement, relative DIC intensities and orientation angles of the gold nanorod. The entire recording is divided into four segments as labeled: (1) searching for a track; (2) vertical transport; (3) a big turn; (4) lateral transport with twisted up-and-down motions. b The 3D trajectory of the cargo. The starting position $(0,0,0)$ is highlighted by the red star. c Parallax-DIC image of the 5D-SPT of the gold nanorod of interest as indicated by the red boxes

by a 20-s quiet period (Fig. 3f and Supplementary Movie 5). During the quiet period, the nanorod displayed a nearly constant bright intensity but slow changes in the dark intensity from 542 to $553.7 \mathrm{~s}$. At $553.7 \mathrm{~s}$, the DIC image of the nanorod changed from completely dark to completely bright within only 2-3 frames (less than $100 \mathrm{~ms}$ ), indicating the nanorod suddenly restored active rotational diffusion. The translational motion of the vesicle followed at $559.9 \mathrm{~s}$, showing a slow moving away from the original entry spot. At $570.2 \mathrm{~s}(\sim 16.5 \mathrm{~s}$ after the detachment event), we observed that the clathrin coat already disassembled from the vesicle (Supplementary Movie 6). This nanorodcontaining vesicle was continuously followed until it was actively transported inside the cell at $\sim 599.4 \mathrm{~s}$ (Supplementary Movie 7).

Intracellular transport in 3D cellular environments. Following the endocytosis, the endocytic vesicles can be transported intracellularly by motor proteins on the microtubules and actin filaments. Within the first $\sim 45 \mathrm{~min}$ following the cellular uptake, the endocytosed substances mostly reside in early endosomes that are about $50-100 \mathrm{~nm}$ in diameter ${ }^{47,48}$. As discussed extensively in our previous publication ${ }^{22}$, the transferrin-coated gold nanorods are bound to the membrane receptors and tightly wrapped by the vesicle membranes. These nanorods generally lose the independent rotational freedoms relative to the vesicles; therefore, they can be excellent reporters of translational and rotational motions of early endosomes being transported by motor proteins.

In the present study, the $5 \mathrm{D}-\mathrm{SPT}$ technique enabled us to reveal the full motions of nanorod-containing vesicles in living A549 cells with unprecedented accuracy. It should be noted that in order to track the movement in the $z$-axis, the automatic feedback control was always activated when studying intracellular transport. This was different from the intermittent activation when studying endocytosis, during which the nanorods were in or on the membrane with little vertical movement other than focus drift.

One representative example is shown in Fig. 4 and Supplementary Movie 8 . The random but confined rotational motions of the cargo within the first second of the recording likely reflect the process of the cargo searching for a cytoskeleton track. Starting at $\sim 1.0 \mathrm{~s}$, the cargo moved nearly straight along the $z$-axis for $\sim 300 \mathrm{~nm}$ and returned at $2.1 \mathrm{~s}$, suggesting a possible back-andforth movement along a track that is perpendicular to the image plane. Then the cargo made a big turn during a pause lasting from 2.1 to $2.9 \mathrm{~s}$, followed by lateral movement of over $5 \mu \mathrm{m}$ in the last $2 \mathrm{~s}$ of the recording. The big turn was likely due to the cargo switching from the perpendicular track to a horizontal one. The cargo also underwent a fast reversal (going back and forth) at $3.0 \mathrm{~s}$, while the rotational behaviors of the nanorod could be observed, possibly because of the rearrangement of motors during which the tension on the cargo was weakened ${ }^{22}$. These observations combine to render the process of cargo transport inside a living cell with great details: cargo searching for a track, directional transport, track-switching, and pause. Three additional examples are provided in Supplementary Figs. 11-13 and Supplementary Movies 9-12.

An interesting sequence of movement was observed at $3.6-5.0 \mathrm{~s}$ in Fig. 4, when the cargo changed its moving direction laterally and vertically, accompanied by changes in its orientation. The sinusoidal-shaped vertical displacements were in the range of 
$100-160 \mathrm{~nm}$, in good agreement with the span of the $80 \mathrm{~nm}$ long gold nanorod when it tumbled around a microtubule track of $\sim 25 \mathrm{~nm}$ in diameter. Combined with the nearly linear lateral ( $x$ and $y$ ) trajectories, a likely explanation is that the cargo moves along the microtubule track with a twister-like motion. This phenomenon may be ascribed to microtubule bending (a curved conformation of the tubulin dimers $)^{49}, 50$. Although the steric hindrance induced by the crowded cellular environment is another plausible explanation, the smoothness of the $3 \mathrm{D}$ trajectory suggests a low probability of a completely random maneuver. This type of motion in live cells is not attainable with other SPT methods.

\section{Discussion}

The findings in the current study disclose characteristic rotational motions of nanorod cargos at different endocytic stages: active rotation, quiet period, and active rotation again with diffusion and transportation. Combining with the dynamic clathrin aggregation observed in fluorescence mode, this rotation-based model not only supports the current endocytosis models derived from the spatial and temporal organization of modular protein molecule groups ${ }^{51-57}$, but also provides new insights in the understanding of endocytosis progression. In the early phase of the modular model, the coating module-proteins involved in cargo recruitment and coat formation-forms on the membrane. This can be confirmed in our fluorescence observations that clathrin assembles at this early stage. Further, the 5D-SPT technique reveals that in this phase, the loss of translational freedom of the cargo does not coincide with the loss of rotational freedom. The nanorod kept rotating after apparent clathrin aggregation proves that the complete fixation of the cargo on the cell membrane happens after the initiation of the endocytosis process. The rotational motion eventually comes to a complete stop, which may be attributed to three factors: (1) the clathrin coat wrapping around the CCP to greatly reduce the fluidity of lipid membranes; (2) the polymerization of actin filaments to anchor the CCP; and (3) the polymerization of dynamin at the neck of the CCP to fully encapsulate the nanorod inside the CCP. Further confirmation of the roles of actin and dynamin is currently underway. The 5D-SPT technique provides a unique opportunity to study the functions of different assisting proteins and how different coatings facilitate or inhibit endocytosis.

On the other hand, we demonstrated the direct visualization of the full motions of the cargo in the $3 \mathrm{D}$ cellular environment during the intracellular transport. Future efforts along this line may provide new evidence to complete our understanding on the competition and coordination of kinesin and dynein motor proteins in live cells. Furthermore, a unique trait of the 5D-SPT technique is that the recorded moving traces reflect the $3 \mathrm{D}$ organization of the cytoskeleton tracks inside the cell. Although the current set-up does not allow the visualization of the 3D structures of the cytoskeleton, it is promising that the 5D tracking of intracellular transport can be combined with super-resolution imaging of cytoskeleton structures ${ }^{58}$ so that the transport mechanism of cargos can be better elucidated. Critical future steps are to combine the 5D-SPT of plasmonic nanoparticle probes with the fluorescence imaging of associated proteins, such as tau ${ }^{59-61}$ and dynactin 62 , to understand the regulated intracellular transport at the molecular level.

In summary, the parallax-DIC microscope and accompanying tracking program have been developed to realize automatic $5 \mathrm{D}-\mathrm{SPT}$ in live cells. The axial movement of gold nanorod probe is detected by monitoring the distance between two half-plane images generated with a wedge prism. A vertical localization precision of $17 \mathrm{~nm}$ has been achieved. As demonstrated through the live cell SPT experiments, the $3 \mathrm{D}$ trajectories and $\mathrm{DIC}$ intensities incorporating rotational information of gold nanorodcontaining vesicles can be correlated to elucidate different stages of the CME and the intracellular transport events in unprecedented detail, which makes the 5D-SPT technique a promising tool to shed new light on the working mechanisms of molecular motors in live cells.

\section{Methods}

Imaging system. The Nikon Eclipse 80i upright microscope used in the present study supports DIC mode and epifluorescence mode. The new parallax-DIC mode was enabled by adding a custom-cut wedge prism $\left(0.5^{\circ}\right.$ wedge angle deviation, Edmund, Barrington, NJ) (Fig. 2) in the light path.

When the microscope was operated in epifluorescence mode, the sample was excited by a mercury lamp. When the microscope was operated in DIC mode, a set of Nomarski DIC optics (including two Nomarski prisms, two polarizers, and a quarter-wave plate) were used. A halogen lamp was used to illuminate the samples through an oil immersion condenser (numerical aperture (NA) 1.40) and the optical signals were collected with a 100× Plan Apo VC NA 1.40 oil immersion objective. The DIC images at a selected wavelength were collected by inserting the corresponding bandpass filter (Semrock, Rochester, NY) into the light path in the microscope.

In parallax-DIC mode, the wedge prism set was placed at the back focal plane of the objective in the DIC configuration. The addition of the wedge prism split the light in half, resulting in the formation of two half-plane images. Details of the implementation and evaluation of the 5D-SPT technique are presented in the Supplementary Note 1.

A Photometrics Evolve 512 EMCCD camera was used for image and video recordings. A rotary motor from Sigma Koki (model no. SGSP-60YAM) was coupled to the microscope stage to control the $z$-position of the sample. A heating stage was used when necessary. The 5D-SPT technique was implanted by compiling the correlation mapping and $z$ calibration into a NIH ImageJ/ $\mu$ Manager ${ }^{63,64}$ plugin using Java. MATLAB and NIH ImageJ were used to analyze the collected images and videos.

Preparation of transferrin-coated gold naonrods. Gold nanorods used in the experiments were all obtained from Nanopartz (Salt Lake City, UT). The purchased gold nanorods were stabilized in cetyltrimethylammonium bromide solution. Gold nanorods with aspect ratio range from 1.36 to 3 were tested and the optimal sizes were used for the experiments as specified. To functionalize the surface of gold nanorods with transferrin (CAS: 11096-37-0, Sigma-Aldrich), a $N$-hydroxylsuccinimide polyethylene glycol thiol (NHS-PEG-thiol) linker (CAS: 947601-981 , Sigma-Aldrich) was used by following a published protocol ${ }^{65}$. The NHS-PEGthiol linker has both disulfide and succinimidyl functionalities for chemisorption onto gold and covalent coupling of transferrin and/or other proteins. As for the gold nanorods, excessive surfactant was first removed from $1.0 \mathrm{~mL}$ gold nanorod solution by centrifugation at $2000 \times g$ for $10 \mathrm{~min}$ and resuspended in $18.2 \mathrm{M} \Omega \mathrm{Milli}$ $\mathrm{Q}$ water. The freshly prepared gold nanorod solution was then mixed with $10 \mu \mathrm{L}$ 20-mM NHS-PEG-thiol solution (in dimethyl sulfoxide) and reacted for $1.5 \mathrm{~h}$. After that, the solution was centrifuged at $2000 \times \mathrm{g}$ for $10 \mathrm{~min}$ and resuspended in Milli-Q water to remove excess NHS-PEG-thiol. An aliquot of $20 \mu \mathrm{L}$ of $2 \mathrm{mg} \mathrm{ml}^{-1}$ transferrin in phosphate buffer saline (PBS, $\mathrm{pH}$ 7.4) was added to the gold nanorod solution and reacted for $3 \mathrm{~h}$. The transferrin-coated gold nanorods were then centrifuged at $2000 \times g$ for $10 \mathrm{~min}$ and resuspended in Milli-Q water before use.

Cell culture for live cell imaging. A549 human lung cancer cells (CCL-185, ATCC) were plated in a T25 cell culture flask (Corning, NY) and grown in complete cell culture medium containing F-12K medium (30-2004, ATCC) and $10 \%$ fetal bovine serum (FBS, 30-2020, ATCC) in a cell culture incubator $\left(37^{\circ} \mathrm{C}\right.$, $5 \% \mathrm{CO}_{2}$ ). An appropriate amount of cell suspension solution was subcultured onto a $22 \times 22 \mathrm{~mm}$ poly-L-lysine (PLL)-coated coverslip, housed in a $35-\mathrm{mm}$ Petri dish (Corning, NY) and kept in the cell culture incubator for $1 \mathrm{~h}$ to allow the cells to attach to the coverslip. After that, $1.5 \mathrm{~mL}$ complete cell culture medium was added to immerse the coverslip. The cells were ready for use when the cell culture reached about $70 \%$ confluency. Two pieces of double-sided tape served as spacers were attached on top of a pre-cleaned glass slide. A coverslip with cells was placed on top of the tapes with two edges to form a chamber, with the cell side facing the glass slide. The two open sides of the chamber were sealed by nail polish to prevent evaporation. The sandwiched chamber was made right before imaging.

Transfection of A549 cell to express clathrin-LCa-EYFP. E. Coli bacteria with clathrin-LCa-EYFP plasmid was acquired from Addgene (Plasmid 21741, Cambridge, MA ${ }^{41}$. The plasmid was extracted from the bacteria by using plasmid extraction kit (cat. 27104) and the corresponding protocol from QIAGEN (Valencia, CA). The A549 cells were pre-grown on coverslip in a Petri dish for $24 \mathrm{~h}$ in the incubator. The EYFP-clathrin plasmid was mixed with the transfection reagent (FuGene6, Roche Applied Science, Indianapolis, IN) in serum-free cell 
culture media first and introduced into the Petri dish containing the cells. After additional 24-48 $\mathrm{h}$ incubation, the cells were ready to use. In control experiments, the cells were incubated with hypertonic media ( $0.45 \mathrm{M}$ Sucrose) or clathrin coat disruption drug $\left(10 \mu \mathrm{g} \mathrm{ml}^{-1}\right.$ Chlorpromazine Chloride) at $37^{\circ} \mathrm{C}$ before exposure to the surface-modified gold nanorods.

siRNA knockdown. The siRNA oligonucleotide sequence 5'-GAGCAUGUGCAC GCUGGCCA- $3^{\prime}$ targeting $\mu 2-2$ and the control siRNA oligonucleotide sequence 5'-AACACAGCAACCUCUACUUGG-3' were both purchased from Dharmacon, Inc. through custom synthesis option. The siRNA knockdown was performed by using oligofectamine (Invitrogen) following the manufacturer's instructions. Briefly, A549 cells were plated onto $35-\mathrm{mm}$ Petri dishes 1 day before transfection in growth medium without antibiotics so that cells would be $30-50 \%$ confluent at the time of transfection. For each dish, $3 \mu \mathrm{L}$ oligofectamine was diluted and mixed gently by pipetting up and down in Opti-MEM ${ }^{\circledast}$ I Medium (Life Technologies) to a final volume $15 \mu \mathrm{L}$ and incubated for $10 \mathrm{~min}$ at room temperature, while $5 \mu \mathrm{L}$ of a $20 \mu \mathrm{M}$ stock oligonucleotide ( $10 \mathrm{nmol}$ synthetic siRNA in $500 \mu \mathrm{L}$ of $1 \times$ siRNA buffer) was diluted and mixed gently in Opti-MEM ${ }^{\circledast}$ I Medium to a total volume of $185 \mu \mathrm{L}$. Then the two solutions were mixed gently and incubated at room temperature for $30 \mathrm{~min}$. This mixture was then combined with $800 \mu \mathrm{L}$ of Opti-MEM ${ }^{\circledast}$ I Medium and added to the cells after washing once with Opti-MEM ${ }^{\otimes}$ I Medium. The final concentration of siRNA was $100 \mathrm{nM}$. An aliquot of $500 \mu \mathrm{L}$ of growth medium without antibiotics was added after incubating the cells with the transfection mixture in the cell incubator for $4 \mathrm{~h}$. The cells were kept in the incubator until they were trypsinized the next day. Second transfection was then performed following the same protocol as described above 1 day after replating. Cells were trypsinized $48 \mathrm{~h}$ after the second transfection, replated on $22 \times 22 \mathrm{~mm}$ coverslips and allowed to grow one additional day for DIC microscopy experiments.

Disruption of AP-2-dependant endocytosis of transferrin-coated gold nanorods after siRNA treatment were assessed by DIC microscopy. Cells were transfected and treated as described above. An aliquot of $25 \mu \mathrm{L}$ of $0.05 \mathrm{nM}$ transferrin-coated gold nanorod $(40 \times 80 \mathrm{~nm})$ solution was mixed into $1 \mathrm{~mL}$ serum-free medium and added to the cells after washing cells with $1 \times \mathrm{PBS}$ and incubated at $37^{\circ} \mathrm{C}$ in the cell incubator for $30 \mathrm{~min}$. The cells were then washed three cycles with cold $1 \times$ PBS and fixed for $10 \mathrm{~min}$ at room temperature in freshly prepared formaldehyde solution containing $3 \%$ paraformaldehyde and $0.1 \%$ glutaraldehyde, followed by three cycles of 10-min washing with $1 \times$ PBS on a shaker. The uptake of gold nanorod was visualized under a DIC microscope at different wavelengths and $z$-stacks were acquired using the objective scanner and reconstructed in Amira, a 3D visualization and analysis program.

Data availability. All relevant data are available from the authors on request.

Received: 22 February 2017 Accepted: 9 August 2017

Published online: 12 October 2017

\section{References}

1. Stender, A. S. et al. Single cell optical imaging and spectroscopy. Chem. Rev. 113, 2469-2527 (2013).

2. Yildiz, A. et al. Myosin V walks hand-over-hand: single fluorophore imaging with 1.5-nm localization. Science 300, 2061-2065 (2003).

3. Pertsinidis, A., Zhang, Y. \& Chu, S. Subnanometre single-molecule localization, registration and distance measurements. Nature 466, 647-651 (2010).

4. Yildiz, A. \& Selvin, P. R. Fluorescence imaging with one manometer accuracy: application to molecular motors. Acc. Chem. Res. 38, 574-582 (2005).

5. Ram, S., Prabhat, P., Chao, J., Ward, E. S. \& Ober, R. High accuracy 3D quantum dot tracking with multifocal plane microscopy for the study of fast intracellular dynamics in live cells. Biophys. J. 95, 6025-6043 (2008).

6. Prabhat, P., Ram, S., Ward, E. S. \& Ober, R. J. Simultaneous imaging of different focal planes in fluorescence microscopy for the study of cellular dynamics in three dimensions. IEEE Trans. Nanobiosci. 3, 237-242 (2004).

7. Speidel, M., Jonas, A. \& Florin, E. L. Three-dimensional tracking of fluorescent nanoparticles with subnanometer precision by use of off-focus imaging. Opt. Lett. 28, 69-71 (2003).

8. Toprak, E., Balci, H., Blehm, B. H. \& Selvin, P. R. Three-dimensional particle tracking via bifocal imaging. Nano Lett. 7, 2043-2045 (2007).

9. Juette, M. F. et al. Three-dimensional sub-100 nm resolution fluorescence microscopy of thick samples. Nat. Methods 5, 527-529 (2008).

10. Pavani, S. R. P., DeLuca, J. G. \& Piestun, R. Polarization sensitive, threedimensional, single-molecule imaging of cells with a double-helix system. Opt. Express 17, 19644-19655 (2009)

11. Ajo-Franklin, C. M., Ganesan, P. V. \& Boxer, S. G. Variable incidence angle fluorescence interference contrast microscopy for z-imaging single objects. Biophys. J. 89, 2759-2769 (2005).
12. Sun, W., Marchuk, K., Wang, G. F. \& Fang, N. Autocalibrated scanning-angle prism-type total internal reflection fluorescence microscopy for nanometerprecision axial position determination. Anal. Chem. 82, 2441-2447 (2010).

13. Marchuk, K., Guo, Y., Sun, W., Vela, J. \& Fang, N. High-precision tracking with non-blinking quantum dots resolves nanoscale vertical displacement. J. Am. Chem. Soc. 134, 6108-6111 (2012).

14. Huang, B., Wang, W., Bates, M. \& Zhuang, X. Three-dimensional superresolution imaging by stochastic optical reconstruction microscopy. Science 319, 810-813 (2008).

15. Yajima, J., Mizutani, K. \& Nishizaka, T. A torque component present in mitotic kinesin Eg 5 revealed by three-dimensional tracking. Nat. Struct. Mol. Biol. 15, 1119-1121 (2008).

16. Sun, Y., McKenna, J. D., Murray, J. M., Ostap, E. M. \& Goldman, Y. E. Parallax: high accuracy three-dimensional single molecule tracking using split images. Nano Lett. 9, 2676-2682 (2009).

17. Welsher, K. \& Yang, H. Multi-resolution 3D Visualisation of the early stages of cellular uptake of peptide-coated nanoparticles. Nat. Nanotechnol. 9, 198-203 (2014).

18. Perillo, E. P. et al. Deep and high-resolution 3D tracking of single particles using nonlinear and multiplexed illumination. Nat. Commun. 6, 7874 (2015).

19. Willets, K. A. \& Van Duyne, R. P. Localized surface plasmon resonance spectroscopy and sensing. Annu. Rev. Phys. Chem. 58, 267-297 (2007).

20. Wang, G., Sun, W., Luo, Y. \& Fang, N. Resolving rotational motions of nano-objects in engineered environments and live cells with gold nanorods and differential interference contrast microscopy. J. Am. Chem. Soc. 132, 16417-16422 (2010)

21. Gu, Y., Sun, W., Wang, G. \& Fang, N. Single particle orientation and rotation tracking discloses distinctive rotational dynamics of drug delivery vectors on live cell membranes. J. Am. Chem. Soc. 133, 5720-5723 (2011).

22. $\mathrm{Gu}, \mathrm{Y}$. et al. Rotational dynamics of cargos at pauses during axonal transport. Nat. Commun. 3, 1030 (2012).

23. $\mathrm{Gu}$, Y. et al. Revealing rotational modes of functionalized gold nanorods on live cell membranes. Small 9, 785-792 (2013).

24. Hopt, A. \& Neher, E. Highly nonlinear photodamage in two-photon fluorescence microscopy. Biophys. J. 80, 2029-2036 (2001).

25. Sonnichsen, C. \& Alivisatos, A. P. Gold nanorods as novel nonbleaching plasmon-based orientation sensors for polarized single-particle microscopy. Nano Lett. 5, 301-304 (2005).

26. Xiao, L., Wei, L., Liu, C., He, Y. \& Yeung, E. S. Unsynchronized translational and rotational diffusion of nanocargo on a living cell membrane. Angew. Chem Int. Ed. 51, 4181-4184 (2012).

27. Spetzler, D. et al. Microsecond time scale rotation measurements of single F-1-ATPase molecules. Biochemistry 45, 3117-3124 (2006).

28. Li, T. et al. Three-dimensional orientation sensors by defocused imaging of gold nanorods through an ordinary wide-field microscope. ACS Nano 6, 1268-1277 (2012).

29. Chang, W.-S., Ha, J. W., Slaughter, L. S. \& Link, S. Plasmonic nanorod absorbers as orientation sensors. Proc. Natl Acad. Sci. USA 107, 2781-2786 (2010).

30. Eghtedari, M. et al. High sensitivity of in vivo detection of gold nanorods using a laser optoacoustic imaging system. Nano Lett. 7, 1914-1918 (2007).

31. Fixler, D. \& Zalevsky, Z. In vivo tumor detection using polarization and wavelength reflection characteristics of gold nanorods. Nano Lett. 13, 6292-6296 (2013).

32. Wang, $\mathrm{H}$. et al. In vitro and in vivo two-photon luminescence imaging of single gold nanorods. Proc. Natl Acad. Sci. USA 102, 15752-15756 (2005).

33. Aguet, F., Geissbühler, S., Märki, I., Lasser, T. \& Unser, M. Super-resolution orientation estimation and localization of fluorescent dipoles using 3-D steerable filters. Opt. Express 17, 6829-6848 (2009).

34. Gans, R. The state of ultramicroscopic silver particles. Annalen Der Physik 47, 270-U214 (1915).

35. Sun, W., Wang, G. F., Fang, N. \& Yeung, E. S. Wavelength-dependent differential interference contrast microscopy: selectively imaging nanoparticle probes in live cells. Anal. Chem. 81, 9203-9208 (2009).

36. Pluta, M. in Advanced Light Microscopy Vol. 2. Specialized Methods Ch. 7, 146-197 (Elsevier, 1989).

37. Gelles, J., Schnapp, B. J. \& Sheetz, M. P. Tracking kinesin-driven movements with nanometre-scale precision. Nature 331, 450-453 (1988).

38. Gu, Y., Di, X., Sun, W., Wang, G. \& Fang, N. Three-dimensional superlocalization and tracking of single gold nanoparticles in cells. Anal. Chem. 84, 4111-4117 (2012).

39. Qian, Z. M., Li, H. Y., Sun, H. Z. \& Ho, K. Targeted drug delivery via the transferrin receptor-mediated endocytosis pathway. Pharmacol. Rev. 54, 561-587 (2002).

40. Chithrani, B. D. \& Chan, W. C. W. Elucidating the mechanism of cellular uptake and removal of protein-coated gold nanoparticles of different sizes and shapes. Nano Lett. 7, 1542-1550 (2007). 
41. Chen, C. \& Zhuang, X. W. Epsin 1 is a cargo-specific adaptor for the clathrin-mediated endocytosis of the influenza virus. Proc. Natl Acad. Sci. USA 105, 11790-11795 (2008).

42. Daukas, G. \& Zigmond, S. H. Inhibition of receptor-mediated but not fluidphase endocytosis in polymorphonuclear leukocytes. J. Cell Biol. 101, 1673-1679 (1985)

43. Kam, N. W. S., Liu, Z. A. \& Dai, H. J. Carbon nanotubes as intracellular transporters for proteins and DNA: An investigation of the uptake mechanism and pathway. Angew. Chem. Int. Ed. 45, 577-581 (2006).

44. Motley, A., Bright, N. A., Seaman, M. N. \& Robinson, M. S. Clathrin-mediated endocytosis in AP-2-depleted cells. J. Cell Biol. 162, 909-918 (2003).

45. Mettlen, M., Loerke, D., Yarar, D., Danuser, G. \& Schmid, S. L. Cargo- and adaptor-specific mechanisms regulate clathrin-mediated endocytosis. J. Cell Biol. 188, 919-933 (2010).

46. Moody, P. R. et al. Receptor crosslinking: a general method to trigger internalization and lysosomal targeting of therapeutic receptor:ligand complexes. Mol. Ther. 23, 1888-1898 (2015).

47. Gruenberg, J., Griffiths, G. \& Howell, K. E. Characterization of the early endosome and putative endocytic carrier vesicles in vivo and with an assay of vesicle fusion in vitro. J. Cell Biol. 108, 1301-1316 (1989).

48. Murk, J. et al. Endosomal compartmentalization in three dimensions: implications for membrane fusion. Proc. Natl Acad. Sci. USA 100, 13332-13337 (2003).

49. Brangwynne, C. P., MacKintosh, F. C. \& Weitz, D. A. Force fluctuations and polymerization dynamics of intracellular microtubules. Proc. Natl Acad. Sci. USA 104, 16128-16133 (2007)

50. Grafmüller, A. \& Voth, G. A. Intrinsic bending of microtubule protofilaments. Structure 19, 409-417 (2011).

51. Yarar, D., Waterman-Storer, C. M. \& Schmid, S. L. A dynamic actin cytoskeleton functions at multiple stages of clathrin-mediated endocytosis. Mol. Biol. Cell 16, 964-975 (2004).

52. Roth, M. G. Clathrin-mediated endocytosis before fluorescent proteins. Nat. Rev. Mol. Cell Biol. 7, 63-68 (2006).

53. Kaksonen, M., Toret, C. P. \& Drubin, D. G. A modular design for the clathrinand actin-mediated endocytosis machinery. Cell 123, 305-320 (2005).

54. Ayscough, K. R. Defining protein modules for endocytosis. Cell 123, 188-190 (2005).

55. Conner, S. D. \& Schmid, S. L. Regulated portals of entry into the cell. Nature 422, 37-44 (2003)

56. Doherty, G. J. \& McMahon, H. T. Mechanisms of endocytosis. Annu. Rev. Biochem. 78, 857-902 (2009).

57. Robertson, A. S., Smythe, E. \& Ayscough, K. R. Functions of actin in endocytosis. Cell. Mol. Life Sci. 66, 2049-2065 (2009).

58. Bálint, Š., Vilanova, I. V., Álvarez, Á. S. \& Lakadamyali, M. Correlative live-cell and superresolution microscopy reveals cargo transport dynamics at microtubule intersections. Proc. Natl Acad. Sci. USA 110, 3375-3380 (2013).

59. Stamer, K., Vogel, R., Thies, E., Mandelkow, E. \& Mandelkow, E. M. Tau blocks traffic of organelles, neurofilaments, and APP vesicles in neurons and enhances oxidative stress. J. Cell Biol. 156, 1051-1063 (2002).

60. Mandelkow, E. M., Stamer, K., Vogel, R., Thies, E. \& Mandelkow, E. Clogging of axons by tau, inhibition of axonal traffic and starvation of synapses. Neurobiol. Aging 24, 1079-1085 (2003).
61. Dixit, R., Ross, J. L., Goldman, Y. E. \& Holzbaur, E. L. F. Differential regulation of dynein and kinesin motor proteins by tau. Science 319, 1086-1089 (2008). 62. Schroer, T. A. Dynactin. Annu. Rev. Cell Dev. Biol. 20, 759-779 (2004).

63. Schneider, C. A., Rasband, W. S. \& Eliceiri, K. W. NIH image to ImageJ: 25 years of image analysis. Nat. Methods 9, 671-675 (2012).

64. Edelstein, A., Amodaj, N., Hoover, K., Vale, R. \& Stuurman, N. in Current Protocols in Molecular Biology 92:14.20.91-14.20.17 (Wiley, 2010)

65. Narayanan, R., Lipert, R. J. \& Porter, M. D. Cetyltrimethylammonium bromidemodified spherical and cube-like gold nanoparticles as extrinsic Raman labels in surface-enhanced Raman spectroscopy based heterogeneous immunoassays. Anal. Chem. 80, 2265-2271 (2008).

\section{Acknowledgements}

The instrument development was supported by National Science Foundation (CBET-1604612). The study of endocytosis was supported by the National Institution of Health (R01GM115763). T.X. was supported by National Natural Science Foundation of China (61571202) and the Junior Thousand Talents Program of China.

\section{Author contributions}

K.C., Y.G., W.S., B.D., G.W. and N.F. designed and performed the experiments. K.C., Y.G., X.F., T.X. and N.F. designed and realized the auto-feedback control system. All authors analyzed the data and wrote the manuscript.

\section{Additional information}

Supplementary Information accompanies this paper at doi:10.1038/s41467-017-01001-9.

Competing interests: The authors declare no competing financial interests.

Reprints and permission information is available online at http://npg.nature.com/ reprintsandpermissions/

Publisher's note: Springer Nature remains neutral with regard to jurisdictional claims in published maps and institutional affiliations.

\begin{abstract}
(c) (i) Open Access This article is licensed under a Creative Commons Attribution 4.0 International License, which permits use, sharing, adaptation, distribution and reproduction in any medium or format, as long as you give appropriate credit to the original author(s) and the source, provide a link to the Creative Commons license, and indicate if changes were made. The images or other third party material in this article are included in the article's Creative Commons license, unless indicated otherwise in a credit line to the material. If material is not included in the article's Creative Commons license and your intended use is not permitted by statutory regulation or exceeds the permitted use, you will need to obtain permission directly from the copyright holder. To view a copy of this license, visit http://creativecommons.org/ licenses/by/4.0/.
\end{abstract}

(C) The Author(s) 2017 